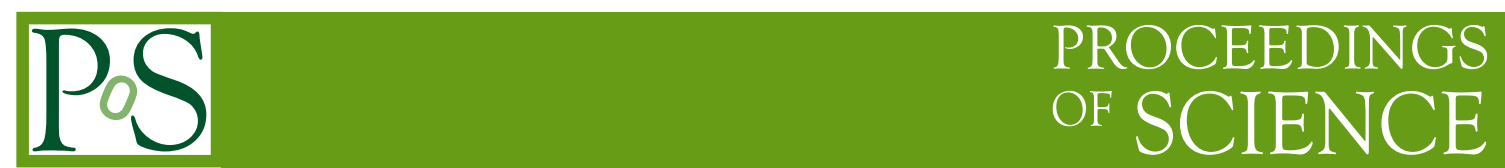

\title{
An introduction to quantum sheaf cohomology
}

\author{
Eric Sharpe* \\ Physics Department \\ Robeson Hall (0435) \\ Virginia Tech \\ Blacksburg, VA 24061 \\ E-mail: ersharpe@vt.edu
}

In this short note, we give a brief introduction to $(0,2)$ mirror symmetry and quantum sheaf cohomology, oriented towards an audience of young physicists. After reviewing general aspects of $(0,2)$ mirrors, we review the A model topological field theory and A/2 (pseudo-)topological field theory, and work through the details of computations of some products of sheaf cohomology classes in simple cases in pedagogically-oriented detail. We also give general results for A/2 correlation functions on $\mathbb{P}^{1} \times \mathbb{P}^{1}$, and discuss global aspects of $\mathrm{A} / 2$ model correlation functions over $(0,2)$ moduli spaces.

7th Conference Mathematical Methods in Physics - Londrina 2012,

16 to 20 April 2012

Rio de Janeiro, Brazil

* Speaker. 


\section{Introduction}

Quantum sheaf cohomology is a generalization of quantum cohomology that appears in $(0,2)$ mirror symmetry. Ordinary mirror symmetry is a duality in which pairs of (typically topologicallydistinct) spaces $X, X^{o}$ are described by the same superconformal field theory:

$$
\operatorname{CFT}(X)=\operatorname{CFT}\left(X^{o}\right) .
$$

One of the original motivations for interest in mirror symmetry was that it could be used to make predictions for Gromov-Witten invariants (counts of minimal-area curves) in the Calabi-Yau's. Physically, those minimal-area curves are instantons in the two-dimensional quantum field theory, which make a nonperturbative contribution to operator products. As there is a correspondence between chiral primary fields in these superconformal field theories and cohomology of the target spaces $X, X^{o}$, the effect of those instanton contributions is to give OPE's which have the structure of a deformation of the cohomology rings, and for this reason the resulting OPE rings are known as "quantum cohomology."

$(0,2)$ mirror symmetry is an analogous duality that arises in perturbative heterotic string compactifications. To specify a heterotic superconformal field theory (which will have $(0,2)$ supersymmetry, hence the name), one specifies ${ }^{1}$ a complex Kähler Calabi-Yau manifold $X$ together with a holomorphic vector bundle $\mathscr{E} \rightarrow X$, satisfying the "Green-Schwarz" anomaly cancellation condition

$$
\operatorname{ch}_{2}(T X)=\operatorname{ch}_{2}(\mathscr{E})
$$

We say that two valid pairs $(X, \mathscr{E}),\left(X^{o}, \mathscr{E}^{o}\right)$ are $(0,2)$-mirror-symmetric if they define the same $(0,2)$ superconformal field theory:

$$
\operatorname{CFT}(X, \mathscr{E})=\operatorname{CFT}\left(X^{o}, \mathscr{E} o\right) .
$$

Just as nonperturbative effects deform OPE rings in $(2,2)$ supersymmetric theories, so too in the $(0,2)$ case do OPE rings get nonperturbative corrections. In the $(0,2)$ case, however, those rings are classically computed by bundle-valued differential forms ("sheaf cohomology"), hence the nonperturbatively-deformed version is known as quantum sheaf cohomology, which will be the focus of much of this review.

$(0,2)$ mirror symmetry was first studied in the mid-90s, see for example $[3,4,5,6,7,8,9]$. It was revived more recently in [10], who made a prediction for quantum sheaf cohomology for certain bundles on $\mathbb{P}^{1} \times \mathbb{P}^{1}$. A suitable corresponding notion of quantum sheaf cohomology was worked out in [11], and the example of [10] was verified. Since then, quantum sheaf cohomology has been further explored in a number of papers, including $[12,13,14,15,16,17,18,19,20,21$, $22,23,24]$ and reviewed in e.g. [25, 26, 27, 28, 29].

In this paper we will briefly review some aspects of quantum sheaf cohomology. A number of reviews have previously appeared describing formal aspects of its computation (definition of suitable induced bundles, formal aspects of computations), so instead this review will focus on pedagogically outlining basics and computational details which have not appeared elsewhere.

\footnotetext{
${ }^{1}$ More general constructions exist, see for example [1,2] for a pair of examples of different types of generalizations. In this review article we will focus on the simpler case of Kähler Calabi-Yau manifolds and holomorphic vector bundles.
} 
We begin with a brief review of the A model topological field theory, and its $(0,2)$ cousin, the A/2 model. After briefly outlining how quantum sheaf cohomology is defined formally, we work through the details of computing pertinent sheaf cohomology groups in simple examples on $\mathbb{P}^{1} \times \mathbb{P}^{1}$. We then state the general result for correlation functions of arbitrary tangent bundle deformations on $\mathbb{P}^{1} \times \mathbb{P}^{1}$, and also outline some aspects of how quantum sheaf cohomology varies globally over a $(0,2)$ moduli space. We conclude with a discussion of open problems and future directions.

\section{Review of the A model topological field theory}

The A model topological field theory is defined by 'twists' of the nonlinear sigma model, a theory with Lagrangian

$$
\frac{1}{\alpha^{\prime}} \int_{\Sigma} d^{2} z\left(\left(g_{\mu \nu}+i B_{\mu \nu}\right) \partial \phi^{\mu} \bar{\partial} \phi^{v}+\frac{i}{2} g_{\mu \nu} \psi_{+}^{\mu} D_{\bar{z}} \psi_{+}^{v}+\frac{i}{2} g_{\mu \nu} \psi_{-}^{\mu} D_{z} \psi_{-}^{v}+R_{i \bar{\jmath} k \bar{l}} \psi_{+}^{i} \psi_{+}^{\bar{\jmath}} \psi_{-}^{k} \psi_{-}^{\bar{l}}\right),
$$

where $\phi: \Sigma \rightarrow X$ is a map from the worldsheet $\Sigma$ into the space $X$ in which the string propagates, and the $\psi_{ \pm}^{\mu}$ are fermionic superpartners of the coordinates $\phi^{\mu}$ on $X$. This theory possesses four supersymmetries, two right-moving, two left-moving, which act as follows:

$$
\begin{aligned}
\delta \phi^{i} & =i \alpha_{-} \psi_{+}^{i}+i \alpha_{+} \psi_{-}^{i}, \\
\delta \phi^{\bar{\imath}} & =i \tilde{\alpha}_{-} \psi_{+}^{\bar{\imath}}+i \tilde{\alpha}_{+} \psi_{-}^{\bar{\imath}}, \\
\delta \psi_{+}^{i} & =-\tilde{\alpha}_{-} \partial \phi^{i}-i \alpha_{+} \psi_{-}^{j} \Gamma_{j m}^{i} \psi_{+}^{m}, \\
\delta \psi_{+}^{\bar{\imath}} & =-\alpha_{-} \partial \phi^{\bar{\imath}}-i \tilde{\alpha}_{+} \psi_{-}^{\bar{\jmath}} \Gamma_{\bar{J}}^{\bar{l}} \psi_{+}^{\bar{m}}, \\
\delta \psi_{-}^{i} & =-\tilde{\alpha}_{+} \bar{\partial} \phi^{i}-i \alpha_{-} \psi_{+}^{j} \Gamma_{j m}^{i} \psi_{-}^{m}, \\
\delta \psi_{-}^{\bar{\imath}} & =-\alpha_{+} \bar{\partial} \phi^{\bar{\imath}}-i \tilde{\alpha}_{-} \psi_{+}^{\bar{j}} \Gamma_{\bar{\jmath}}^{\bar{l}} \psi_{-}^{\bar{m}} .
\end{aligned}
$$

The four supersymmetry transformation parameters are $\alpha_{ \pm}, \tilde{\alpha}_{ \pm}$. Since there is one pair of each chirality, a theory with this set of supersymmetry transformations is said to possess " $(2,2)$ " supersymmetry.

The A model is obtained by a subtle change in the definition of the fermions $\psi_{ \pm}^{i, \bar{l}}$. Specifically, in the A model, we take them to be worldsheet scalars and vectors, defined technically as follows [30]:

$$
\begin{array}{ll}
\psi_{+}^{i}\left(\equiv \psi_{z}^{i}\right) \in \Gamma_{C^{\infty}}\left(K \otimes \phi^{*} T^{1,0} X\right), & \psi_{-}^{i}\left(\equiv \chi^{i}\right) \in \Gamma_{C^{\infty}}\left(\left(\phi^{*} T^{0,1} X\right)^{*}\right), \\
\psi_{+}^{\bar{\tau}}\left(\equiv \chi^{\bar{\imath}}\right) \in \Gamma_{C^{\infty}}\left(\left(\phi^{*} T^{1,0} X\right)^{*}\right), & \psi_{-}^{\bar{\tau}}\left(\equiv \psi_{\bar{z}}^{\bar{\tau}}\right) \in \Gamma_{C^{\infty}}\left(\bar{K} \otimes \phi^{*} T^{0,1} X\right) .
\end{array}
$$

This is a fancy way of saying that $\psi_{+}^{i}, \psi_{-}^{\bar{\imath}}$ are worldsheet vectors, and $\psi_{+}^{\bar{i}}, \psi_{-}^{i}$ are worldsheet scalars. The Lagrangian is unchanged (except that the worldsheet spin connection terms in the covariant derivatives in the fermion kinetic terms are modified).

The supersymmetry transformation parameters also necessarily rotate into worldsheet scalars and vectors at the same time. The supersymmetry parameters which become scalars are $\alpha_{-}$and $\tilde{\alpha}_{+}$, which we shall rename $\alpha$ and $\tilde{\alpha}$ for the rest of this section. If we restrict to those supersymmetry transformations, we get

$$
\delta \phi^{i}=i \alpha \chi^{i}
$$




$$
\begin{aligned}
\delta \phi^{\bar{\imath}} & =i \tilde{\alpha} \chi^{\bar{\imath}}, \\
\delta \chi^{i} & =0, \\
\delta \chi^{\bar{\imath}} & =0, \\
\delta \psi_{z}^{\bar{\imath}} & =-\alpha \partial \phi^{\bar{\imath}}-i \tilde{\alpha} \chi^{\bar{\jmath}} \Gamma_{\bar{\jmath} m}^{\bar{l}} \psi_{z}^{\bar{m}}, \\
\delta \psi_{\bar{z}}^{i} & =-\tilde{\alpha} \bar{\partial} \phi^{i}-i \alpha \chi^{j} \Gamma_{j m}^{i} \psi_{\bar{z}}^{m} .
\end{aligned}
$$

It is straightforward to check that these transformations are nilpotent.

Now, nilpotent scalar symmetries play an important role in quantum field theory. They arise prototypically in gauge-fixing, and because of their application there, they are known in general as BRST transformations. The operator generating such transformations (in this case, defined by $\alpha=\tilde{\alpha}$ ) is commonly denoted $Q$, and known as the BRST operator. In the context of gauge theories, the physical states are taken to be states which are annihilated by $Q$, said to be "BRST closed" or $Q$ closed, modulo states in the image of $Q$, said to be "BRST exact" or " $Q$ exact", and the ghost and gauge-fixing terms one adds to the Lagrangian are BRST exact. As a result, if one deforms the gauge-fixing condition, for example, the result is to deform the lagrangian by a BRST exact term, which cannot modify correlation functions of physical states (which are annihilated by $Q$ ). In other words, formally, if the operators $\mathscr{O}_{1} \cdots \mathscr{O}_{n}$ are BRST closed, then

$$
\left\langle\mathscr{O}_{1} \cdots \mathscr{O}_{n} \exp (Q(-))\right\rangle=\left\langle\mathscr{O}_{1} \cdots \mathscr{O}_{n}\right\rangle
$$

In the case of the A model topological field theory, it can be shown that the entire action is BRST exact. Hence, changing the value of $\alpha^{\prime}$ is equivalent to adding or subtracting copies of the (BRST exact) action, and so, from the same argument as above, correlation functions of BRST closed states are necessarily unaffected. As a result, such correlation functions can be computed at any convenient value of $\alpha^{\prime}$, and one typically chooses $\alpha^{\prime}=0$, for which choice all Feynman diagram contributions to correlation functions are turned off ${ }^{2}$.

Now, let us consider which states of this theory are BRST closed. Since the $\chi$ 's are annihilated by the BRST operator, such states should be of the form

$$
b_{i_{1} \cdots i_{n} \bar{J}_{1} \cdots \bar{J}_{m}} \chi^{i_{1}} \cdots \chi^{i_{n}} \chi^{\bar{\jmath}_{1}} \cdots \chi^{\bar{J}_{m}} .
$$

Looking at the form of the BRST action above, we see that there is a simple dictionary we can apply to understand how to count BRST closed states of the form above. Specifically, if we identify

$$
\chi^{i} \sim d z^{i}, \chi^{\bar{J}} \sim d \bar{z}^{\bar{J}}
$$

then since $Q \phi \propto \chi$, we see that we can usefully identify $Q$ with the exterior derivative $d$ on differential forms. Thus, BRST closed forms of the form above should be identified with $d$-closed differential forms

$$
b_{i_{1} \cdots i_{n} \bar{\jmath}_{1} \cdots \bar{\jmath}_{m}} d z^{i_{1}} \wedge \cdots \wedge d z^{i_{n}} \wedge d \bar{z}^{\bar{J}_{1}} \wedge \cdots \wedge d \bar{z}^{\bar{J}_{m}}
$$

and so are counted by de Rham cohomology groups.

Since Feynman diagrams do not contribute to correlation functions of BRST-closed operators, correlation functions reduce to zero mode computations.

\footnotetext{
${ }^{2}$ Except involving special cases in which the factors of $\alpha^{\prime}$ cancel out.
} 
Consider, for example, the special case that the target space is $\mathbb{P}^{n}$. In this case, classical contributions to correlation functions will have the form

$$
\left\langle\mathscr{O}_{1} \cdots \mathscr{O}_{m}\right\rangle=\int_{\mathbb{P} n} \omega_{1} \wedge \cdots \wedge \omega_{m}
$$

where $\omega_{i}$ is the differential form corresponding to the operator $\mathscr{O}_{i}$, the integral over $\mathbb{P}^{n}$ arises as the integral over the space of bosonic zero modes inside the path integral, and the ordinary Grassmann integrals over fermi zero modes have soaked up all of the $\chi$ 's in the $\mathscr{O}$ 's. Thus, we see the classical contribution will be nonzero if and only if the wedge product of the corresponding differential forms is a top-form.

In an instanton sector of degree $d$, containing maps of degree $d$ from the worldsheet to $\mathbb{P}^{n}$, contributions will have an analogous form ${ }^{3}$ :

$$
\left\langle\mathscr{O}_{1} \cdots \mathscr{O}_{m}\right\rangle_{d}=\int_{\mathscr{M}_{d}} \tilde{\omega}_{1} \wedge \cdots \wedge \tilde{\omega}_{m}
$$

where $\mathscr{M}_{d}$ is the moduli space of instantons of degree $d$, and $\tilde{\omega}_{i}$ is the differential form on $\mathscr{M}$ corresponding to the operator $\mathscr{O}_{i}$. (Note as a special case, $\mathscr{M}_{0}$ is the same as the target space, in this case $\mathbb{P}^{n}$.) Each instanton sector is weighted by $q=\exp \left(-\int J+i \int B\right)$, where $J$ is the Kähler form, so that the complete correlation function can be (schematically) written in the form

$$
\left\langle\mathscr{O}_{1} \cdots \mathscr{O}_{m}\right\rangle=\sum_{d} q^{d}\left\langle\mathscr{O}_{1} \cdots \mathscr{O}_{m}\right\rangle_{d} .
$$

Now, implicitly above we have assumed that the moduli space $\mathscr{M}$ is both smooth and compact, but typically neither will be true. Nevertheless, there exist standard methods for compactifying instanton moduli spaces, and in the present case, for $\mathbb{P}^{n}$, one useful choice of compactification yields

$$
\mathscr{M}_{d}=\mathbb{P}^{(n+1)(d+1)-1} .
$$

We are now in a position to derive quantum cohomology for $\mathbb{P}^{n}$. Let $\omega$ denote the generator of the classical cohomology ring of $\mathbb{P}^{n}$, a two-form, and let $\mathscr{O}$ denote the corresponding operator. Then from the fact that the integrals above will be nonzero only when they are integrating a top-form, we find that nonzero correlation functions are of the form

$$
\left\langle\mathscr{O}^{(n+1)(d+1)-1}\right\rangle_{d} \propto q^{d}
$$

in instanton sector $d$, where $q=\exp \left(-\int J+i \int B\right), J$ the Kähler form, $B$ the two-form gauge field. Writing

$$
\mathscr{O}^{(n+1)(d+1)-1}=\mathscr{O}^{n} \mathscr{O}^{d(n+1)},
$$

we see that the correlation functions are equivalently encoded by the OPE relation

$$
\mathscr{O}^{n+1}=q
$$

which looks like a deformation of the standard classical cohomology ring relation in $\mathbb{P}^{n}$, namely $\omega^{n+1}=0$. The OPE ring in the A model defines what is called the "quantum cohomology" ring.

\footnotetext{
${ }^{3}$ For reasons of brevity, we are ignoring the effects of the four-fermi term, which generate a factor of the top Chern class of an 'obstruction' bundle when there are fermion vector zero modes.
} 


\section{The $A / 2$ model}

The $\mathrm{A} / 2$ model is a twist of the $(0,2)$ nonlinear sigma model

$$
\frac{1}{\alpha^{\prime}} \int_{\Sigma} d^{2} z\left(\left(g_{\mu v}+i B_{\mu v}\right) \partial \phi^{\mu} \bar{\partial} \phi^{v}+\frac{i}{2} g_{\mu \nu} \psi_{+}^{\mu} D_{\bar{z}} \psi_{+}^{v}+\frac{i}{2} h_{\alpha \beta} \lambda_{-}^{\alpha} D_{z} \lambda_{-}^{\beta}+F_{i \bar{j} \bar{b}} \psi_{+}^{i} \psi_{+}^{\bar{\jmath}} \lambda_{-}^{a} \lambda_{-}^{\bar{b}}\right),
$$

The twist is defined by taking the fermions to couple to bundles as follows:

$$
\begin{array}{lll}
\psi_{+}^{i} \in \Gamma_{C^{\infty}}\left(K \otimes \phi^{*} T^{1,0} X\right), & \lambda_{-}^{a} \in \Gamma_{C^{\infty}}\left(\phi^{*} \overline{\mathscr{E}}^{*}\right), \\
\psi_{+}^{\bar{i}} \in \Gamma_{C^{\infty}}\left(\left(\phi^{*} T^{1,0} X\right)^{*}\right), & \lambda_{-}^{\bar{a}} \in \Gamma_{C^{\infty}}\left(\bar{K} \otimes \phi^{*} \overline{\mathscr{E}}\right) .
\end{array}
$$

This is a close analogue of the A model twist, and in fact, in the special case that $\mathscr{E}=T X$, the $\mathrm{A} / 2$ model becomes the A model. It is called $(0,2)$ because it has only the right-moving half of the supersymmetry of the $(2,2)$ supersymmetric model of the previous section.

Because this is a chiral theory, there are potential anomalies. To cancel anomalies in the original physical theory, one imposes the Green-Schwarz condition

$$
\operatorname{ch}_{2}(\mathscr{E})=\operatorname{ch}_{2}(T X)
$$

In addition, in order for the $\mathrm{A} / 2$ model to be well-defined, one must impose the additional constraint that

$$
\wedge^{\text {top }} \mathscr{E}^{*} \cong K_{X}
$$

(This is a consequence of the necessity to make the Grassmann-zero-mode integrals inside the path integral well-defined.) For example, when $\mathscr{E}=T X$, both of these constraints are satisfied trivially.

The A/2 model contains a BRST operator acting as

$$
\delta \phi^{i}=0, \delta \phi^{\bar{\imath}} \propto \psi_{+}^{\bar{\imath}}, \delta \psi_{+}^{\bar{i}}=0=\delta \lambda_{-}^{a}, \delta \psi_{+}^{i} \neq 0, \delta \lambda_{-}^{\bar{a}} \neq 0 .
$$

The states of the $\mathrm{A} / 2$ model generalizing the A model states are of the form

$$
b_{\bar{l}_{1} \cdots \bar{l}_{q} a_{1} \cdots a_{p}} \psi_{+}^{\bar{l}_{1}} \cdots \psi_{+}^{\bar{l}_{q}} \lambda_{-}^{a_{1}} \cdots \lambda_{-}^{a_{p}} .
$$

Proceeding in an analogous fashion, the BRST operator $Q$ is identified with $\bar{\partial}$, and the states above are identified with bundle-valued differential forms, counted by sheaf cohomology $H^{q}\left(X, \wedge^{p} \mathscr{E}^{*}\right)$.

Unlike the ordinary A model, here we do not have a true topological field theory, since the BRST symmetry only acts on the right-movers. For this reason, this structure is occasionally termed a 'holomorphic field theory.'

We can approximate correlation functions in the $\mathrm{A} / 2$ model by truncating to zero mode computations. Then, correlation functions can be described in a fashion that is formally very similar to the A model. For example, classical contributions to correlation functions are of the form

$$
\left\langle\mathscr{O}_{1} \cdots \mathscr{O}_{m}\right\rangle=\int_{X} \omega_{1} \wedge \cdots \wedge \omega_{m}
$$

where $X$ is the target space (= space of bosonic zero modes, classically), and $\omega_{i}$ is a bundle-valued differential form (a representative of sheaf cohomology) corresponding to the operator $\mathscr{O}_{i}$. 
If

$$
\omega_{i} \in H^{p}\left(X, \wedge^{q} \mathscr{E}^{*}\right)
$$

then the integral above will be nonzero when the integrand is an element of

$$
H^{\text {top }}\left(X, \wedge^{\text {top }} \mathscr{E}^{*}\right)
$$

(That much, at least, is guaranteed physically by selection rules on the right- and left-moving global $U(1)$ symmetries on the worldsheet.) To extract a number, we must use the additional constraint that $\wedge^{\text {top }} \mathscr{E}^{*} \cong K_{X}$. Using that isomorphism, we can write the integrand as an element of

$$
H^{\text {top }}\left(X, K_{X}\right)
$$

which can be integrated to get a number.

In an instanton sector of degree $d$, for simplicity ignoring four-fermi terms, contributions to correlation functions follow the same form as we have seen for the A model:

$$
\left\langle\mathscr{O}_{1} \cdots \mathscr{O}_{m}\right\rangle_{d}=\int_{\mathscr{M}_{d}} \tilde{\omega}_{1} \cdots \wedge \tilde{\omega}_{m}
$$

where $\mathscr{M}_{d}$ is the instanton moduli space of degree $d$ (= space of bosonic zero modes), and $\tilde{\omega}_{i}$ is the element of sheaf cohomology on $\mathscr{M}_{d}$ corresponding to the operator $\mathscr{O}_{i}$.

Now, let us examine the expression above more closely. If

$$
\omega_{i} \in H^{p}\left(X, \wedge^{q} \mathscr{E}^{*}\right)
$$

then

$$
\tilde{\omega}_{i} \in H^{p}\left(\mathscr{M}_{d}, \wedge^{q} \mathscr{F}^{*}\right)
$$

where $\mathscr{F}$ is the (induced) sheaf of left-moving fermi zero modes over $\mathscr{M}_{d}$. (Mathematically, if there is a universal instanton $\alpha$, this is $R^{0} \pi_{*} \alpha^{*} \mathscr{E}$.) The integrand of the expression for a correlation function in a degree $d$ sector is then an element of

$$
H^{\text {top }}\left(\mathscr{M}_{d}, \wedge^{\text {top }} \mathscr{F}^{*}\right)
$$

Formally (from index theory), if both $\wedge^{\text {top }} \mathscr{E}^{*} \cong K_{X}$ and $\operatorname{ch}_{2}(T X)=\operatorname{ch}_{2}(\mathscr{E})$, then ${ }^{4} \wedge^{\text {top } \mathscr{F}} \cong K_{\mathscr{M}}$, which can then be integrated to get a number.

It should now be clear that to actually perform these computations, to make sense of the A/2 theory, we not only need to compactify the moduli space, we must also make sense of $\mathscr{F}$, and extend it over the compactification, in such a way that $\wedge^{\text {top } \mathscr{F}^{*}} \cong K_{\mathscr{M}}$. Procedures for constructing both moduli spaces and induced sheaves with all desired properties have been described in reviews elsewhere, we shall not repeat them in detail here.

For toric varieties, if $\mathscr{E}$ is a deformation of the tangent bundle, then $\mathscr{M}_{d}$ is a toric variety and $\mathscr{F}$ is a deformation of its tangent bundle determined by $\mathscr{E}$.

\footnotetext{
${ }^{4}$ In a sector with no contribution from four fermi terms. In more general sectors, there are analogous though more complicated expressions. For reasons of both brevity and simplicity, we have elected not to describe the most general case in this short introduction.
} 


\section{Sample computations in a simple example}

Let us now outline how to perform these computations explicitly, in a simple example, following the same procedure as in $[11,14]$ (albeit in an easier special case). The basic manipulations can largely be reduced to linear algebra, once one understands the general procedure, and our goal in this section will be to demonstrate that procedure.

Let us consider $\mathbb{P}^{1} \times \mathbb{P}^{1}$ with a gauge bundle $\mathscr{E}$ determined as the cokernel of

$$
0 \longrightarrow \mathscr{O}^{2} \stackrel{E}{\longrightarrow} \mathscr{O}(1,0)^{2} \oplus \mathscr{O}(0,1)^{2} \longrightarrow \mathscr{E} \longrightarrow 0
$$

where

$$
E=\left[\begin{array}{cc}
x_{1} & \gamma x_{2} \\
x_{2} & 0 \\
0 & \tilde{x_{1}} \\
0 & \tilde{x_{2}}
\end{array}\right]
$$

where $x_{i}, \tilde{x}_{j}$ are homogeneous coordinates on each of the two $\mathbb{P}^{1}$ factors. If $\gamma=0$, this becomes a standard description of the tangent bundle of $\mathbb{P}^{1} \times \mathbb{P}^{1}$, hence this is a simple one-parameter deformation of the tangent bundle.

Let us describe the classical cup products, at the level of Cech cohomology. We will cover $\mathbb{P}^{1} \times \mathbb{P}^{1}$ by open sets

$$
U_{i j}=\left\{x_{i} \neq 0, \tilde{x}_{j} \neq 0\right\} .
$$

First, we will need to find representatives of the sheaf cohomology groups $H^{*}\left(\mathscr{E}^{*}\right)$, in terms of Cech cohomology. Note that by dualizing the sequence above, we get

$$
0 \longrightarrow \mathscr{E}^{*} \longrightarrow \mathscr{O}(-1,0)^{2} \oplus \mathscr{O}(0,-1)^{2} \stackrel{E}{\longrightarrow} \mathscr{O}^{2} \longrightarrow 0
$$

which implies

$$
H^{0}\left(\mathscr{O}(-1,0)^{2} \oplus \mathscr{O}(0,-1)^{2}\right) \longrightarrow H^{0}\left(\mathscr{O}^{2}\right) \longrightarrow H^{1}\left(\mathscr{E}^{*}\right) \longrightarrow H^{1}\left(\mathscr{O}(-1,0)^{2} \oplus \mathscr{O}(0,-1)^{2}\right)
$$

However,

$$
H^{0}\left(\mathscr{O}(-1,0)^{2} \oplus \mathscr{O}(0,-1)^{2}\right)=0=H^{1}\left(\mathscr{O}(-1,0)^{2} \oplus \mathscr{O}(0,-1)^{2}\right),
$$

hence we see that

$$
H^{1}\left(\mathscr{E}^{*}\right) \cong H^{0}\left(\mathscr{O}^{2}\right)
$$

As a result, our strategy for computing representatives of generators of $H^{1}\left(\mathscr{E}^{*}\right)$ will be to explicitly compute the coboundary map acting on elements of a basis for $H^{0}\left(\mathscr{O}^{2}\right)$.

Let $Y, \tilde{Y}$ denote the elements of $H^{1}\left(\mathscr{E}^{*}\right)$ corresponding (via the coboundary isomorphism above) to elements of the two standard basis elements of $H^{0}\left(\mathscr{O}^{2}\right)$. We will let $Y_{i, j ; i^{\prime}, j^{\prime}}, \tilde{Y}_{i, j ; i^{\prime}, j^{\prime}}$ denote the Cech representatives on $U_{i, j} \cap U_{i^{\prime}, j^{\prime}}$, which can be computed as follows.

First, we will need to lift the basis elements of $H^{0}\left(\mathscr{O}^{2}\right)$ to meromorphic sections of

$$
\mathscr{O}(-1,0)^{2} \oplus \mathscr{O}(0,-1)^{2} \text {. }
$$

We will denote the lifts by $L_{i, j}, \tilde{L}_{i, j}$, and then use them to compute $Y, \tilde{Y}$. 
The lifts can be computed as follows. First, consider $U_{1,1}$. Since we are working on the patch in which $x_{1} \neq 0$ and $\tilde{x}_{1} \neq 0$, that means that the lift should be of the form

$$
L_{1,1}=\frac{1}{x_{1} \tilde{x}_{1}}\left[\begin{array}{l}
a \tilde{x}_{1}+b \tilde{x}_{2} \\
c \tilde{x}_{1}+d \tilde{x}_{2} \\
e x_{1}+f x_{2} \\
g x_{1}+h x_{2}
\end{array}\right]
$$

for some constants $a, b, c, d, e, f, g, h$ such that

$$
E^{T} \cdot L_{1,1}=\left[\begin{array}{l}
1 \\
0
\end{array}\right] .
$$

Plugging in, we find this is solved by

$$
L_{1,1}=\frac{1}{x_{1} \tilde{x}_{1}}\left[\begin{array}{c}
\tilde{x}_{1} \\
0 \\
-\gamma x_{2} \\
0
\end{array}\right]
$$

Similarly, it is straightforward to demonstrate that

$$
L_{1,2}=\frac{1}{x_{1} \tilde{x}_{2}}\left[\begin{array}{c}
\tilde{x}_{2} \\
0 \\
0 \\
-\gamma x_{2}
\end{array}\right], L_{2,1}=\frac{1}{x_{2} \tilde{x}_{1}}\left[\begin{array}{c}
0 \\
\tilde{x}_{1} \\
0 \\
0
\end{array}\right], L_{2,2}=\frac{1}{x_{2} \tilde{x}_{2}}\left[\begin{array}{c}
0 \\
\tilde{x}_{2} \\
0 \\
0
\end{array}\right]
$$

and similarly, solving for $\tilde{L}_{i j}$ such that

$$
E^{T} \cdot \tilde{L}_{i, j}=\left[\begin{array}{l}
0 \\
1
\end{array}\right]
$$

yields

$$
\tilde{L}_{1,1}=\frac{1}{x_{1} \tilde{x}_{1}}\left[\begin{array}{c}
0 \\
0 \\
x_{1} \\
0
\end{array}\right], \tilde{L}_{1,2}=\frac{1}{x_{1} \tilde{x}_{2}}\left[\begin{array}{c}
0 \\
0 \\
0 \\
x_{1}
\end{array}\right], \tilde{L}_{2,1}=\frac{1}{x_{2} \tilde{x}_{1}}\left[\begin{array}{c}
0 \\
0 \\
x_{2} \\
0
\end{array}\right], \tilde{L}_{2,2}=\frac{1}{x_{2} \tilde{x}_{2}}\left[\begin{array}{c}
0 \\
0 \\
0 \\
x_{2}
\end{array}\right] .
$$

Now, the Cech representatives $Y_{i, j ; i^{\prime}, j^{\prime}}, \tilde{Y}_{i, j ; i^{\prime}, j^{\prime}}$ are determined from the lifts by

$$
Y_{i, j ; i^{\prime}, j^{\prime}}=L_{i^{\prime}, j^{\prime}}-L_{i, j}, \tilde{Y}_{i, j ; i^{\prime}, j^{\prime}}=\tilde{L}_{i^{\prime}, j^{\prime}}-\tilde{L}_{i, j}
$$

So far, we have computed Cech representatives of the generators of $H^{1}\left(\mathscr{E}^{*}\right)$.

Next, we need to compute cup products of these cohomology classes, to get Cech representatives of $H^{2}\left(\wedge^{2} \mathscr{E} *\right)$.

The representatives of the cup product are formed from the ratio of the minors of a matrix whose columns form the Cech representatives above, to the reduced maximal minors of the 
nullspace of $E$. For example, let us consider $Y \cup Y$. Its representative on $U_{1,1} \cap U_{1,2} \cap U_{2,1}$ is given by computing the minors of the matrix

$$
\left[Y_{1,1 ; 1,2}, Y_{1,2 ; 2,1}\right]=\left[L_{1,2}-L_{1,1}, L_{2,1}-L_{1,2}\right]=\left[\begin{array}{cc}
0 & -x_{1}^{-1} \\
0 & x_{2}^{-1} \\
\gamma x_{2} x_{1}^{-1} \tilde{x}_{1}^{-1} & 0 \\
-\gamma x_{2} x_{1}^{-1} \tilde{x}_{2}^{-1} & 0
\end{array}\right]
$$

which are

$$
0, \frac{\gamma x_{2}}{x_{1}^{2} \tilde{x}_{1}},-\frac{\gamma x_{2}}{x_{1}^{2} \tilde{x}_{2}},-\frac{\gamma}{x_{1} \tilde{x}_{1}}, \frac{\gamma}{x_{1} \tilde{x}_{2}}, 0
$$

On the other hand, the maximal minors of the nullspace of $E$ are given by

$$
0,-x_{2} \tilde{x}_{2}, x_{2} \tilde{x}_{1}, x_{1} \tilde{x}_{2},-x_{1} \tilde{x}_{1},-\gamma x_{2}^{2},
$$

normalized so that there are no homogeneous coordinates in denominators. (In general, we should divide out common factors to form the 'reduced' maximal minors, but in the list of minors above, there are no common factors.)

Taking a ratio of the first two nonzero corresponding terms, we find

$$
(Y \cup Y)_{1,1 ; 1,2 ; 2,1}=-\frac{\gamma}{x_{1}^{2} \tilde{x}_{1} \tilde{x}_{2}} .
$$

(Note that there is an ambiguity here due to coboundaries, so a any of any two corresponding minors will give the result up to coboundaries.)

Continuing in this fashion, representatives can be computed on any triple overlap. Note that to be consistent, the results must satisfy the cocycle condition

$(\delta Y \cup Y)_{1,1 ; 1,2 ; 2,1 ; 2,2}=(Y \cup Y)_{1,2 ; 2,1 ; 2,2}-(Y \cup Y)_{1,1 ; 2,1 ; 2,2}+(Y \cup Y)_{1,1 ; 1,2 ; 2,2}-(Y \cup Y)_{1,1 ; 1,2 ; 2,1}=0$.

Similarly, $(Y \cup \tilde{Y})_{1,1 ; 1,2 ; 2,1}$ is computed from the ratio of the minors of the matrix

$$
\left[Y_{1,1 ; 1,2}, \tilde{Y}_{1,2 ; 2,1}\right]
$$

to the reduced maximal minors of $E$, and $(\tilde{Y} \cup \tilde{Y})_{1,1 ; 1,2 ; 2,1}$ is computed from the ratio of the minors of the matrix

$$
\left[\tilde{Y}_{1,1 ; 1,2}, \tilde{Y}_{1,2 ; 2,1}\right]
$$

to the reduced maximal minors of $E$.

Finally, to get the correlation functions, we must evaluate an integral:

$$
\langle Y Y\rangle=\int_{\mathbb{P}^{1} \times \mathbb{P}^{1}} Y \cup Y .
$$

This is partly defined by the choice of isomorphism $\wedge^{\text {top }} \mathscr{E}^{*} \cong K_{X}$, and so there is an overall phase ambiguity, which becomes important when considering the behavior of correlation functions over the moduli space. In the language of Cech cohomology, we need a trace which extracts pieces proportional to

1

$\overline{\text { product of homogeneous coordinates }}$ 
or in other words, a trace that does not see any coboundary that does not touch every patch. Schematically, if we pick an ordering on coordinate patches, then the trace formula should have the form

$$
\begin{gathered}
\text { (data on } \left.U_{1} \cap U_{2} \cap U_{3}\right)-\left(\text { data on } U_{2} \cap U_{3} \cap U_{4}\right)+\left(\text { data on } U_{3} \cap U_{4} \cap U_{5}\right) \\
-\left(\text { data on } U_{4} \cap U_{5} \cap U_{6}\right)+\cdots
\end{gathered}
$$

and we should normalize by the product of the homogeneous coordinates. In the present case, that means we want

$$
\begin{aligned}
& \langle Y Y\rangle=\left(x_{1} x_{2} \tilde{x}_{1} \tilde{x}_{2}\right)\left((Y \cup Y)_{1,1 ; 1,2 ; 2,1}-(Y \cup Y)_{1,2 ; 2,1 ; 2,2}\right), \\
& \langle Y \tilde{Y}\rangle=\left(x_{1} x_{2} \tilde{x}_{1} \tilde{x}_{2}\right)\left((Y \cup \tilde{Y})_{1,1 ; 1,2 ; 2,1}-(Y \cup \tilde{Y})_{1,2 ; 2,1 ; 2,2}\right), \\
& \langle\tilde{Y} \tilde{Y}\rangle=\left(x_{1} x_{2} \tilde{x}_{1} \tilde{x}_{2}\right)\left((\tilde{Y} \cup \tilde{Y})_{1,1 ; 1,2 ; 2,1}-(\tilde{Y} \cup \tilde{Y})_{1,2 ; 2,1 ; 2,2}\right) .
\end{aligned}
$$

The result may have irrelevant coboundary terms, which (because of our construction of the trace) must be removed manually.

In the present case, the expressions above yield

$$
\begin{aligned}
\langle Y Y\rangle & =-\frac{\gamma x_{2}}{x_{1}}, \\
\langle Y \tilde{Y}\rangle & =1, \\
\langle\tilde{Y} \tilde{Y}\rangle & =0 .
\end{aligned}
$$

The term in $\langle Y Y\rangle$, proportional to $x_{2} / x_{1}$, is an example of a coboundary term which must be removed manually. After doing so, our final result for correlation functions is given by

$$
\begin{aligned}
\langle Y Y\rangle & =0, \\
\langle Y \tilde{Y}\rangle & =1, \\
\langle\tilde{Y} \tilde{Y}\rangle & =0 .
\end{aligned}
$$

This is identical to the classical cohomology ring in the undeformed theory, the special case that $\mathscr{E}$ is the tangent bundle of $\mathbb{P}^{1} \times \mathbb{P}^{1}$. We can identify $Y$ with the volume form on one $\mathbb{P}^{1}$, and $\tilde{Y}$ with the volume form on the second $\mathbb{P}^{1}$; the only way to get a nonzero wedge product is to wedge $Y$ and $\tilde{Y}$ together.

Let us outline how to perform analogous computations in instanton sectors. Consider the sector of instantons of degree $(1,0)$. Here, following earlier remarks, the instanton moduli space will be $\mathbb{P}^{3} \times \mathbb{P}^{1}$, the product of the degree 1 instanton moduli space on one $\mathbb{P}^{1}$ factor and the degree 0 instanton moduli space on the second $\mathbb{P}^{1}$ factor. The induced sheaf $\mathscr{F}$ wil be described as the cokernel

$$
0 \longrightarrow \mathscr{O}^{2} \stackrel{E^{\prime}}{\longrightarrow} \mathscr{O}(1,0)^{4} \oplus \mathscr{O}(0,1)^{2} \longrightarrow \mathscr{F} \longrightarrow 0
$$

where

$$
E^{\prime}=\left[\begin{array}{cc}
x_{11} & \gamma x_{21} \\
x_{12} & \gamma x_{22} \\
x_{21} & 0 \\
x_{22} & 0 \\
0 & \tilde{x}_{1} \\
0 & \tilde{x}_{2}
\end{array}\right]
$$


where $x_{11}, x_{12}, x_{21}, x_{22}$ are homogeneous coordinates on $\mathbb{P}^{3}$, and $\tilde{x}_{1}, \tilde{x}_{2}$ are homogeneous coordinates on the $\mathbb{P}^{1}$ factor. This expression was derived from $E$ in the classical case by expanding homogeneous coordinates there in zero modes:

$$
\begin{aligned}
& x_{1}=x_{11} u+x_{12} v, \\
& x_{2}=x_{21} u+x_{22} v,
\end{aligned}
$$

(where $u, v$ are homogeneous coordinates on the worldsheet, assumed to be a $\mathbb{P}^{1}$ ), then reorganizing by common worldsheet factors, i.e. the first and third lines of $E^{\prime}$ are the coefficients of $u$, the second and fourth lines are the coefficients of $v$, and so forth.

Proceeding in the same fashion as before, one finds

$$
\begin{aligned}
& \langle Y Y Y Y\rangle_{1,0}=0 \\
& \langle Y Y Y \tilde{Y}\rangle_{1,0}=1, \\
& \langle Y Y \tilde{Y} \tilde{Y}\rangle_{1,0}=0 \\
& \langle Y \tilde{Y} \tilde{Y} \tilde{Y}\rangle_{1,0}=0 \\
& \langle\tilde{Y} \tilde{Y} \tilde{Y} \tilde{Y}\rangle_{1,0}=0 .
\end{aligned}
$$

When one repeats in the $(0,1)$ instanton sector, one finds

$$
\begin{aligned}
\langle Y Y Y Y\rangle_{0,1} & =0 \\
\langle Y Y Y \tilde{Y}\rangle_{0,1} & =0 \\
\langle Y Y \tilde{Y} \tilde{Y}\rangle_{0,1} & =0 \\
\langle Y \tilde{Y} \tilde{Y} \tilde{Y}\rangle_{0,1} & =1 \\
\langle\tilde{Y} \tilde{Y} \tilde{Y} \tilde{Y}\rangle_{0,1} & =0 .
\end{aligned}
$$

These can be combined into four-point correlation functions as

$$
\left\langle\mathscr{O}_{1} \mathscr{O}_{2} \mathscr{O}_{3} \mathscr{O}_{4}\right\rangle=q\left\langle\mathscr{O}_{1} \mathscr{O}_{2} \mathscr{O}_{3} \mathscr{O}_{4}\right\rangle_{1,0}+\tilde{q}\left\langle\mathscr{O}_{1} \mathscr{O}_{2} \mathscr{O}_{3} \mathscr{O}_{4}\right\rangle_{0,1}
$$

If one repeats for a few more instanton sectors, one can quickly convince oneself that the operator product relations are defined by

$$
Y^{2}=q, \tilde{Y}^{2}=\tilde{q}
$$

the same as for the undeformed theory.

\section{General correlation functions for $\mathbb{P}^{1} \times \mathbb{P}^{1}$}

Although we will not work through all the details, let us now describe the result for correlation functions for the most general possible tangent bundle deformation on $\mathbb{P}^{1} \times \mathbb{P}^{1}$.

Consider $\mathbb{P}^{1} \times \mathbb{P}^{1}$ with bundle $\mathscr{E}$ defined by

$$
0 \longrightarrow \mathscr{O}^{2} \stackrel{E}{\longrightarrow} \mathscr{O}(1,0)^{2} \oplus \mathscr{O}(0,1)^{2} \longrightarrow \mathscr{E} \longrightarrow 0
$$


where

$$
E=\left[\begin{array}{ll}
A x & B x \\
C \tilde{x} & D \tilde{x}
\end{array}\right]
$$

with $A, B, C, D 2 \times 2$ matrices, and $x, \tilde{x} 2$-component vectors of homogeneous coordinates on $\mathbb{P}^{1} \times \mathbb{P}^{1}$.

Define

$$
\begin{aligned}
\Delta= & (\operatorname{det} A)(\operatorname{det} D)-(\operatorname{det} B)(\operatorname{det} C), \\
\phi= & (\operatorname{det}(A+B))^{2}(\operatorname{det} C)(\operatorname{det} D)+(\operatorname{det}(C+D))^{2}(\operatorname{det} A)(\operatorname{det} B) \\
& -(\operatorname{det}(A+B))(\operatorname{det}(C+D))[(\operatorname{det} A)(\operatorname{det} D)+(\operatorname{det} B)(\operatorname{det} C)] \\
& +\Delta(\operatorname{det}(A+B))(\operatorname{det} D-\operatorname{det} C)+\Delta(\operatorname{det}(C+D))(\operatorname{det} A-\operatorname{det} B) .
\end{aligned}
$$

Then, the it can be shown that the classical correlation functions are given by

$$
\begin{aligned}
& \langle Y Y\rangle=\phi^{-1}[\Delta-(\operatorname{det} D)(\operatorname{det}(A+B))+(\operatorname{det} B)(\operatorname{det}(C+D))], \\
& \langle Y \tilde{Y}\rangle=\phi^{-1} \Delta, \\
& \langle\tilde{Y} \tilde{Y}\rangle=\phi^{-1}[\Delta-(\operatorname{det} A)(\operatorname{det}(C+D))+(\operatorname{det} C)(\operatorname{det}(A+B))] .
\end{aligned}
$$

It is straightforward to show that these obey the classical relations

$$
\langle\operatorname{det}(A Y+B \tilde{Y})\rangle=0=\langle\operatorname{det}(C Y+D \tilde{Y})\rangle,
$$

and furthermore it was shown in e.g. [24] that the quantum sheaf cohomology relations in this case are given by

$$
\langle\operatorname{det}(A Y+B \tilde{Y})\rangle=q,\langle\operatorname{det}(C Y+D \tilde{Y})\rangle=\tilde{q}
$$

\section{Global issues}

Let us make a few remarks in passing concerning global issues on the moduli space of $(0,2)$ theories. In ordinary quantum cohomology, $q$ is a parameter, a coordinate. In $(0,2)$ quantum cohomology, however, $q$ is more complicated, essentially because of the $B$ field implicit in the exponent. In a $(2,2)$ theory, the holonomy of the $B$ field is well-defined, but in a $(0,2)$ theory, because of the Green-Schwarz mechanism, that $B$ field changes across coordinate patches. As a result, $q$ picks up phases across coordinate patches, and is better interpreted as a section of a line bundle.

We can see this structure in our example on $\mathbb{P}^{1} \times \mathbb{P}^{1}$. The moduli space of bundles is (partially) constructed from the data $A, B, C, D$ by taking a $G L(2)^{2}$ quotient, which acts on this data as

$$
\left[\begin{array}{ll}
A & B \\
C & D
\end{array}\right] \mapsto\left[\begin{array}{ll}
P A & P B \\
Q C & Q D
\end{array}\right]
$$

where $(P, Q) \in G L(2)^{2}$, and which leaves $Y, \tilde{Y}$ invariant. Under this action, note that

$$
\operatorname{det}(A Y+B \tilde{Y}) \mapsto(\operatorname{det} P) \operatorname{det}(A Y+B \tilde{Y}), \operatorname{det}(C Y+D \tilde{Y}) \mapsto(\operatorname{det} Q) \operatorname{det}(C Y+D \tilde{Y}) .
$$


For example, this implies that the correlation functions transform as sections of nontrivial bundles over the $(0,2)$ moduli space, which corresponds to worldsheet realizations [31] of e.g. the BaggerWitten line bundle [32]. Furthermore, the quantum sheaf cohomology relations

$$
\langle\operatorname{det}(A Y+B \tilde{Y})\rangle=q,\langle\operatorname{det}(C Y+D \tilde{Y})\rangle=\tilde{q}
$$

are only well-defined globally if under the same $G L(2)^{2}$ action,

$$
q \mapsto(\operatorname{det} P) q, \tilde{q} \mapsto(\operatorname{det} Q) \tilde{q}
$$

which implies that over the moduli space of bundles, $q, \tilde{q}$ transform as sections of line bundles. This reflects the fact that on the worldsheet, the global $G L(2)^{2}$ action above is anomalous, as it only acts on left-moving fields.

\section{Open problems and future directions}

At the moment, techniques exist for computing quantum sheaf cohomology only for toric varieties (generalizations of projective spaces) and deformations of their tangent bundles. To be genuinely useful for compact Calabi-Yau string compactifications, we need techniques for evaluating quantum sheaf cohomology for hypersurfaces in toric varieties, for more general bundles than just deformations of tangent bundles.

In standard Gromov-Witten theory, there are many tricks and techniques for doing more general computations, but most boil down to utilizing the fact that the A model topological field theory is independent of complex structures. A priori, it is not believed that the A/2 model is always independent of complex structures, hence those techniques from Gromov-Witten theory should not be applicable here. Nevertheless, it was argued in [17] that at least in special cases, the A/2 model is independent of complex moduli and certain bundle moduli, so that in those cases, computations can be performed. A deeper understanding of the results of [17] would be desirable, as well as generalizations to more cases.

\section{Acknowledgements}

We would like to thank our many collaborators, including L. Anderson, J. Distler, R. Donagi, J. Gray, J. Guffin, S. Katz, I. Melnikov, B. Ovrut, T. Pantev, and S. Sethi, for useful conversations. This work was partially supported by NSF grant PHY-1068725.

\section{References}

[1] I. Melnikov, C. Quigley, S. Sethi, M. Stern, “Target spaces for chiral gauge theories,” arXiv: 1212.1212

[2] J. Distler, E. Sharpe, "Heterotic compactifications with principal bundles for general groups and general levels," hep-th/ 070124 4, Adv. Theor. Math. Phys. 14 (2010) 335-398.

[3] R. Blumenhagen, R. Schimmrigk, A. Wisskirchen, "The $(0,2)$ exactly solvable structure of chiral rings, Landau-Ginzburg theories, and Calabi-Yau manifolds,” Nucl. Phys. B461 (1996) 460-492, hep-th/9510055. 
[4] R. Blumenhagen, A. Wisskirchen, "Exactly solvable points in the moduli space of heterotic N=2 strings," Mod. Phys. Lett. A11 (1996) 1475-1488, hep-th/9601050.

[5] R. Blumenhagen, A. Wisskirchen, "Exploring the moduli space of $(0,2)$ strings," Nucl. Phys. B475 (1996) 225-243, hep-th/9604140.

[6] R. Blumenhagen, M. Flohr, “Aspects of $(0,2)$ orbifolds and mirror symmetry," Phys. Lett. B404 (1997) 41-48, hep-th/ 9702199.

[7] R. Blumenhagen, “Target space duality for (0,2) compactifications," Nucl. Phys. B513 (1998) 573-590, hep-th/9707198.

[8] R. Blumenhagen, “(0,2) target space duality, CICYs and reflexive sheaves,” Nucl. Phys. B514 (1998) 688-704, hep-th/9710021.

[9] R. Blumenhagen, "Issues of heterotic (0,2) compactifications," Fortsch. Phys. 48 (2000) 37-40, hep-th/9901005.

[10] A. Adams, A. Basu, S. Sethi, “(0,2) duality,” Adv. Theor. Math. Phys. 7 (2004) 865-950, hep-th/0309226.

[11] S. Katz, E. Sharpe, "Notes on certain (0,2) correlation functions," Comm. Math. Phys. 262 (2006) 611-644, hep-th/0406226.

[12] E. Sharpe, "Notes on certain other (0,2) correlation functions," Adv. Theor. Math. Phys. 13 (2009) 33-70, hep-th/0605005.

[13] A. Adams, J. Distler, M. Ernebjerg, “Topological heterotic rings,” Adv. Theor. Math. Phys. 10 (2006) 657=682, hep-th $/ 0506263$.

[14] J. Guffin, S. Katz, "Deformed quantum cohomology and (0,2) mirror symmetry," arXiv: 0710.2354

[15] I. Melnikov, S. Sethi, "Half-twisted (0,2) Landau-Ginzburg models,” JHEP 0803 (2008) 040, arXiv: 0712.1058.

[16] J. McOrist, I. Melnikov, "Half-twisted correlators from the Coulomb branch," JHEP 0804 (2008) 071, arXiv: 0712.3272.

[17] J. McOrist, I. Melnikov, "Summing the instantons in half-twisted linear sigma models," JHEP 0902 (2009) 026, arXiv: 0810.0012.

[18] I. Melnikov, “(0,2) Landau-Ginzburg models and residues,” JHEP 0909 (2009) 118, arXiv : 0902.3908

[19] M. Kreuzer, J. McOrist, I. Melnikov, R. Plesser, “(0,2) deformations of linear sigma models," arXiv: 1001.2104.

[20] J. McOrist, “The revival of (0,2) linear sigma models," Int. J. Mod. Phys. A26 (2011) 1-41, arXiv : 1010.4667

[21] J. McOrist, I. Melnikov, “Old issues and linear sigma models,” arXiv: 1103.1322.

[22] J. Guffin, E. Sharpe, “A-twisted heterotic Landau-Ginzburg models,” J. Geom. Phys. 59 (2009) 1581-1596, arXiv: 0801.3955.

[23] R. Donagi, J. Guffin, S. Katz, E. Sharpe, "A mathematical theory of quantum sheaf cohomology," arXiv: 1110.3751. 
[24] R. Donagi, J. Guffin, S. Katz, E. Sharpe, "Physical aspects of quantum sheaf cohomology for deformations of tangent bundles of toric varieties," arXiv: 1110.3752.

[25] E. Sharpe, "Notes on correlation functions in (0,2) theories," pp. 93-104 in String geometry (Snowbird, 2004), American Math. Soc., Providence, Rhode Island, 2004, hep-th/ 0502064.

[26] J. Guffin, “Quantum sheaf cohomology, a precis,” arXiv: 1101.1305.

[27] E. Sharpe, "An introduction to quantum sheaf cohomology," contribution to the proceedings of the Workshop on Landau-Ginzburg models (Grenoble, France, June 2010), to appear in Annales de l'Institut Fourier.

[28] R. Donagi, J. Guffin, S. Katz, E. Sharpe, “(0,2) quantum cohomology,” pp. 83-103 in String-Math 2011, proceedings of String-Math 2011 (University of Pennsylvania, June 2011), Proc. Symp. Pure Math. 85, American Mathematical Society, Rhode Island, 2012.

[29] I. Melnikov, S. Sethi, E. Sharpe, "Recent developments in $(0,2)$ mirror symmetry," arXiv : 1209.1134 , SIGMA 8 (2012) 068.

[30] E. Witten, "Mirror manifolds and topological field theory," hep-th/9112056, pp. 121-160 in Mirror Symmetry I, ed. S.-T. Yau, International Press, Cambridge, Massachusetts, 1998.

[31] J. Distler, "Notes on N=2 sigma models," hep-th/9212062, pp. 234-256 in String theory and quantum gravity'92 (Trieste, 1992).

[32] E. Witten, J. Bagger, “Quantization of Newton's constant in certain supergravity theories,” Phys. Lett. B115 (1982) 202. 